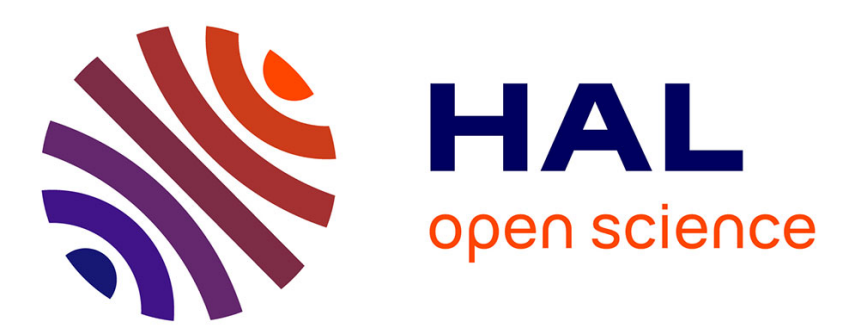

\title{
Nonlinear interactions in a rotating disk flow: From a Volterra model to the Ginzburg-Landau equation
}

\author{
E. Floriani, Thierry Dudok de Wit, P. Le Gal
}

\section{To cite this version:}

E. Floriani, Thierry Dudok de Wit, P. Le Gal. Nonlinear interactions in a rotating disk flow: From a Volterra model to the Ginzburg-Landau equation. Chaos: An Interdisciplinary Journal of Nonlinear Science, 2000, 10 (4), pp.834. 10.1063/1.1285863 . insu-02928474

\section{HAL Id: insu-02928474 https://hal-insu.archives-ouvertes.fr/insu-02928474}

Submitted on 2 Mar 2021

HAL is a multi-disciplinary open access archive for the deposit and dissemination of scientific research documents, whether they are published or not. The documents may come from teaching and research institutions in France or abroad, or from public or private research centers.
L'archive ouverte pluridisciplinaire HAL, est destinée au dépôt et à la diffusion de documents scientifiques de niveau recherche, publiés ou non, émanant des établissements d'enseignement et de recherche français ou étrangers, des laboratoires publics ou privés. 


\section{Nonlinear interactions in a rotating disk flow: From a Volterra model to the Ginzburg- Landau equation}

Cite as: Chaos 10, 834 (2000); https://doi.org/10.1063/1.1285863

Submitted: 29 March 2000 . Accepted: 30 June 2000 . Published Online: 30 November 2000

E. Floriani, T. Dudok de Wit, and P. Le Gal

ARTICLES YOU MAY BE INTERESTED IN

Analysis of von Kármán's swirling flow on a rotating disc in Bingham fluids

Physics of Fluids 28, 013601 (2016); https://doi.org/10.1063/1.4937590

Fluid flow and heat transfer over a rotating and vertically moving disk

Physics of Fluids 30, 063605 (2018); https://doi.org/10.1063/1.5037460

Film flow on a rotating disk

The Physics of Fluids 29, 3522 (1986); https://doi.org/10.1063/1.865829

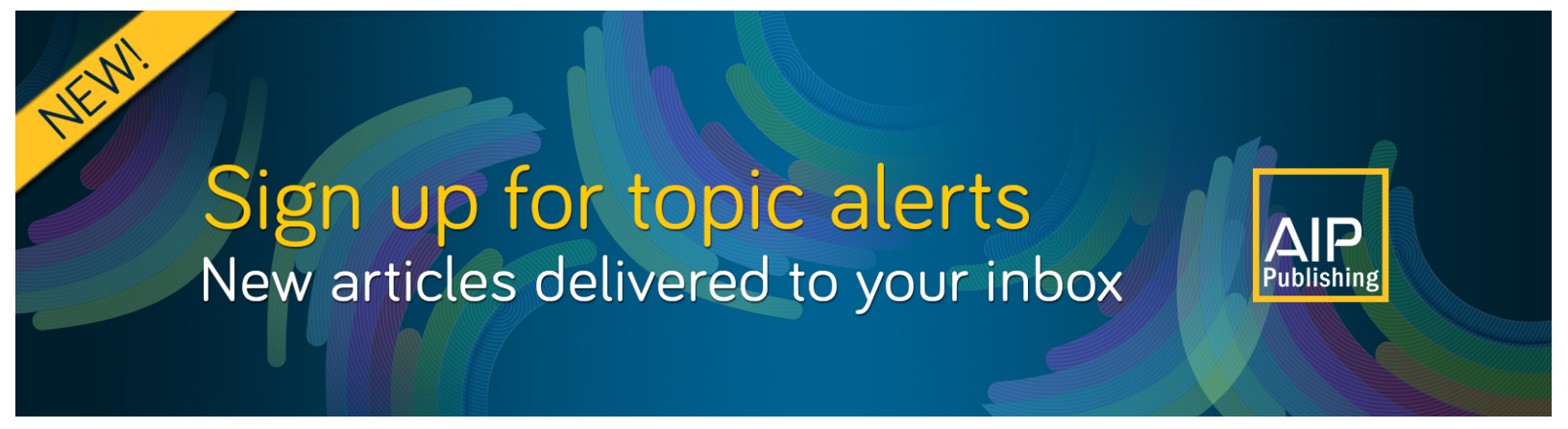




\title{
Nonlinear interactions in a rotating disk flow: From a Volterra model to the Ginzburg-Landau equation
}

\author{
E. Floriania) \\ Centre de Physique Théorique, CNRS Luminy Case 907, 13288 Marseille cedex 9, France \\ T. Dudok de $\mathrm{Wit}^{\text {b) }}$ \\ LPCE-CNRS, 3A, av. de la Recherche Scientifique, 45071 Orléans cedex 2, France \\ P. Le $\mathrm{Gal}^{\mathrm{C})}$ \\ IRPHE, 12, av. du Général Leclerc, 13003 Marseille, France
}

(Received 29 March 2000; accepted for publication 30 June 2000)

\begin{abstract}
The physical system under consideration is the flow above a rotating disk and its cross-flow instability, which is a typical route to turbulence in three-dimensional boundary layers. Our aim is to study the nonlinear properties of the wavefield through a Volterra series equation. The kernels of the Volterra expansion, which contain relevant physical information about the system, are estimated by fitting two-point measurements via a nonlinear parametric model. We then consider describing the wavefield with the complex Ginzburg-Landau equation, and derive analytical relations which express the coefficients of the Ginzburg-Landau equation in terms of the kernels of the Volterra expansion. These relations must hold for a large class of weakly nonlinear systems, in fluid as well as in plasma physics. () 2000 American Institute of Physics. [S1054-1500(00)00204-4]
\end{abstract}

In this paper we analyze the results of a rotating disk flow experiment, using a Volterra series equation. Volterra models can describe a wide variety of nonlinear behavior, with the kernels of the Volterra series carrying the physical information about the system under study; in particular, the higher order kernels represent the intensity of nonlinear interactions between the Fourier components of the wavefield. This approach has mostly been used to characterize nonlinear phenomena in plasmas: here, we explore the possibility of applying the same kind of approach to fluid mechanics, and present a procedure for extracting Volterra kernels from experimental two-point measurements of the fluid velocity. We then translate our results in terms of a Ginzburg-Landau model, which is extensively used in fluid dynamics to describe instabilities and transition to turbulence. More precisely, we show that the coefficients of the GinzburgLandau equation can be analytically calculated as functions of the kernels of the Volterra series equation. This gives an original method of estimating the GinzburgLandau coefficients from experimental data, which is a notoriously difficult task, especially when the system is well beyond the instability threshold.

\section{INTRODUCTION}

Volterra series equations were first applied to the study of nonlinear systems by Wiener ${ }^{1}$ and can be used to describe a large variety of weakly nonlinear behavior. ${ }^{2,3}$ Recently, this

\footnotetext{
${ }^{a)}$ Electronic mail: floriani@cpt.univ-mrs.fr

b)Electronic mail: ddwit@cnrs-orleans.fr

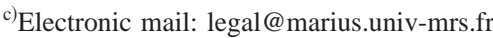

approach has mostly been used for modeling nonlinear interactions and transition to turbulence in plasmas. ${ }^{4,5}$ In this paper, we apply the same type of analysis to a fluid system: we use a Volterra equation, whose kernels will be estimated using two-point measurements of the wavefield, to discuss some properties of the flow in the boundary layer above a rotating disk. ${ }^{6,7}$

Our main aim in this article is to examine the relation existing between the Volterra model and the complex Ginzburg-Landau (GL) amplitude equation, which is one of the classical tools used to describe the instability of flows and their transition to turbulence. ${ }^{8,9}$ We will indeed show that, if a GL equation is applicable, then its coefficients can be analytically calculated as functions of the kernels of the Volterra series equation.

We stress that our approach is not restricted to the GL equation or to a particular physical system, but holds for a large class of weakly nonlinear systems (described, for example, by the nonlinear Schrödinger equation, or the SwiftHohenberg equation), under some general hypotheses that will be specified in the following.

This way of establishing a quantitative link between the experimental signal and the GL model, apart from its theoretical interest, also has a practical importance, since the identification of coefficients of the GL equation from finite and noise corrupted data is notoriously difficult. Volterra models can handle a much larger class of nonlinearities than those described by the GL equation, so our approach can also be used to test if such an amplitude equation is actually applicable to a particular physical system. This last point is important since many experiments have been set up for measuring parameters of the GL equation, without actually providing efficient means for validating the use of such a model. 
The paper is organized as follows: in Sec. II we will present the main features of rotating disk flow instabilities, and briefly describe the experiment that produced the data we analyze here. In Sec. III we will define the Volterra model and discuss its physical interpretation. Section IV is devoted to numerical aspects of model identification while a first overview of the results obtained with the rotating disk data is given in Sec. V. In Sec. VI we will present the analytical calculation of the coefficients of the complex Ginzburg-Landau equation from Volterra kernels. Finally, the results of Sec. VI are applied to the rotating disk flow in Sec. VII.

\section{ROTATING DISK FLOW INSTABILITIES}

Ekman ${ }^{10}$ was the first in 1905 to formulate the mathematical expression of the rotating velocity field of the atmospheric boundary layer at the terrestrial poles. His analysis was based on the linearization of the fluid motion equations and the search for self-similar solutions. He supposed that the fluid and the disk angular velocities are very close to one another. The solution he wrote takes the form of a spiral, now called the "Ekman Spiral," and is mainly localized in a thin boundary layer near the rotating disk. Later, in 1921, Karman generalized this search for self-similar solutions ${ }^{11}$ to the full nonlinear case of a revolving disk limiting a semiinfinite volume of a fluid initially at rest. But it is only from the sixties that the stability of the Ekman or Karman boundary layers was approached. Faller ${ }^{12}$ presented in 1991 a review of the stability of these flows. Two types of instabilities appear in a generic way. They are called type I and type II instabilities. ${ }^{13}$ Type II instability corresponds to a destabilization by the combined effects of the forces due to the Coriolis and viscous effects. It produces waves which are rolled up in spirals in a contrary direction to the disk rotation. On the other hand, the study of Stuart ${ }^{14}$ shows that type I instability is inviscid and comes from the presence of unstable inflection points in the radial velocity profiles. This instability also produces spiral waves but which are rolled up in the direction of rotation of the disk. A great amount of experimental and analytical work has been devoted to this type I instability which is a paradigm for the study of laminarturbulent transition in three-dimensional boundary layer flows. ${ }^{15-18}$ In particular, one of the most recent analyses of these flows was realized by Lingwood, ${ }^{19}$ who demonstrated that these instabilities experience a transition from convective to absolute regimes at given Reynolds numbers which seem to correspond to the Reynolds numbers where the transition to turbulence is usually observed.

As the characteristic boundary layer thickness is $\delta$ $=\sqrt{\nu / \Omega}$ where $\nu$ is the viscosity of the fluid and $\Omega$ the disk angular velocity, one can define the Reynolds number of the flow at radius $r$ by $\operatorname{Re}=r / \delta=\sqrt{\Omega r^{2} / \nu}$. This parameter will grow as a fluid particle moves away from the center of the disk. The flow near the center is then dominated by viscous effects which damp the disturbances: the zone is occupied by a laminar state. But starting from a critical Reynolds number, ${ }^{6}$ three-dimensional disturbances grow and propagate in the boundary layer. This part of the disk is called the

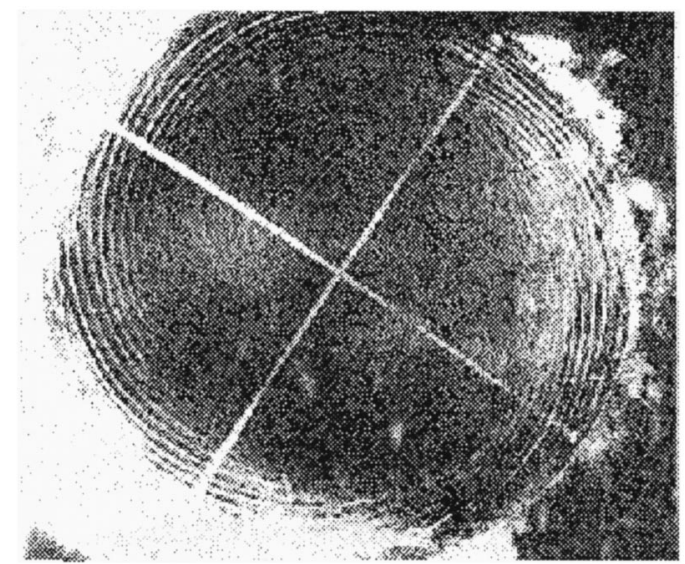

FIG. 1. Type I instability waves in the Karman boundary layer (Ref. 21).

transition zone where more or less quite localized wavepackets ${ }^{20}$ appear and form a crown of vortices at the nonlinear stage of their development. Finally, at the periphery of this zone, the flow is disordered, and presents a turbulent aspect. These three concentric zones are visualized in the illustration of Fig. 1, obtained by dye injection. ${ }^{21}$

The anemometric signals we analyze here come from experiments in which a small roughness element was glued on the disk surface, just under the linear threshold. ${ }^{7}$ A full description of the experimental apparatus is given in Refs. 6 , 7 and the main features of the setup are the following. A stainless steel disk having a diameter of $500 \mathrm{~mm}$, is mounted on a vertical shaft and immersed in a filled up water tank. The disk angular velocity $\Omega$ is fixed at $1 \mathrm{~Hz}$. Above this disk, a circular Plexiglas lid is maintained at a distance of 20 $\mathrm{mm}$ from the disk by a vertical shroud. This cylindrical wall has an inner diameter of $700 \mathrm{~mm}$. Two anemometric probes are plunged vertically through openings practiced in the lid and are positioned at a distance of $0,6 \mathrm{~mm}$ above the disk surface at the desired radial location; they are oriented as shown in Fig. 2. The sensors are directed radially in such a way that the hot films are mainly sensitive to azimuthal velocity fluctuations.

A wave packet of type I instability is generated by the roughness element. Its frequency is centered around $\omega_{c}$ $=32 \mathrm{~Hz}$, and its shape widens as its amplitude grows; see

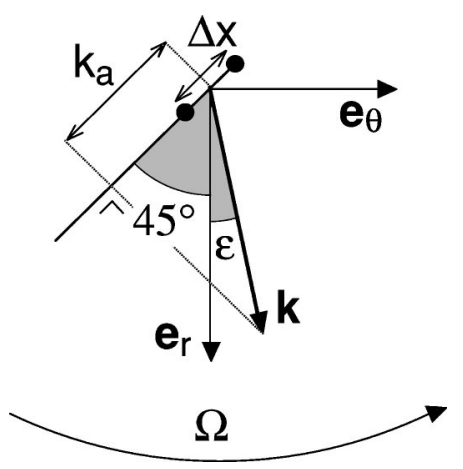

FIG. 2. Orientation of the anemometric probes (marked by dots) with respect to the flow rotation. The wavenumber we measure is the projection $k_{a}$ of $\mathbf{k}$ along $\Delta \mathbf{x}$. In the present experiment, $\Delta x=8 \mathrm{~mm}$ and $\epsilon \approx 18 \mathrm{deg}$. 

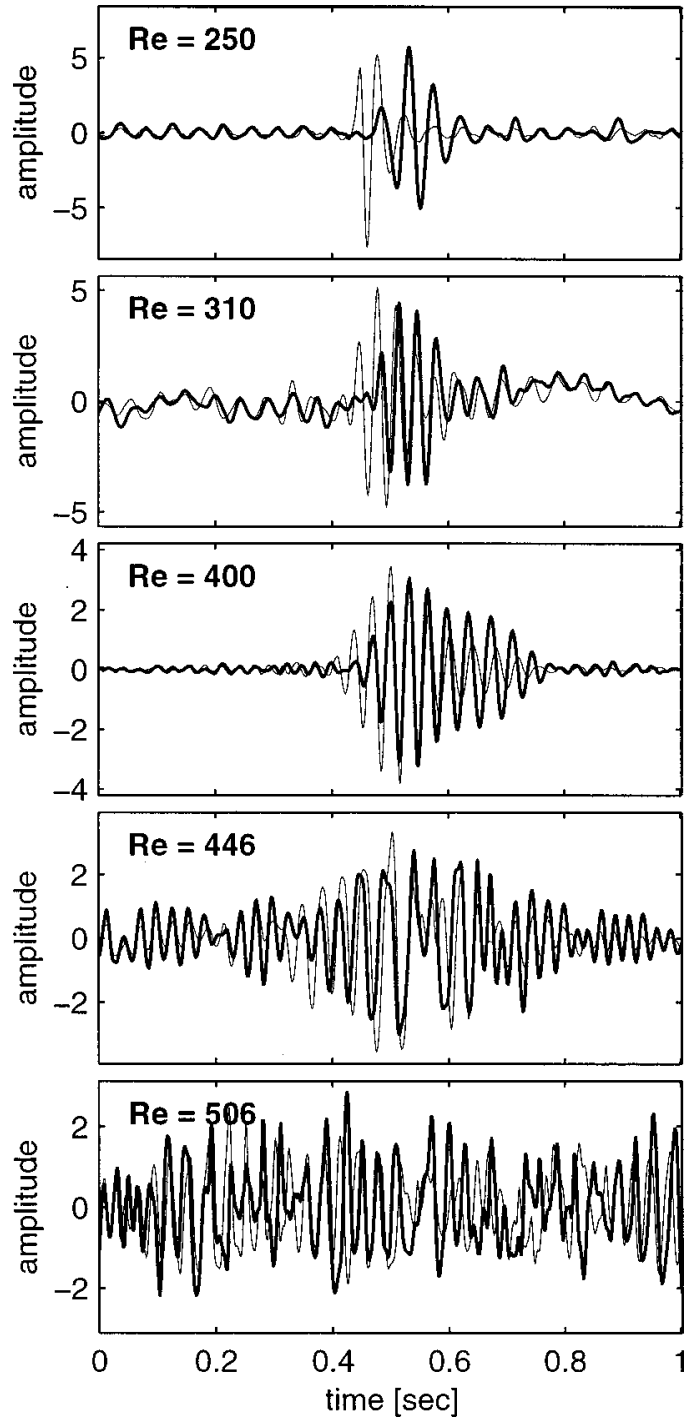

FIG. 3. Excerpt of the wavefield amplitude as measured by the two probes, for different Reynolds numbers. The downstream probe signal is in bold, and amplitude units are arbitrary. The roughness element is approximately located at $0.4 \mathrm{sec}$. See also Fig. 4 of Ref. 7.

Fig. 3. The critical Reynolds number has been found ${ }^{7}$ to be about $\mathrm{Re} \approx 280$, while developed turbulence takes over at $\mathrm{Re} \approx 500$.

As presented in Ref. 7, finite amplitude effects affect the growth and the dynamics of these waves. In particular, it was qualitatively shown that a one-dimensional complex Ginzburg-Landau equation could model some experimental features such as the growth of harmonic modes or the frequency shift due to finite amplitude of the waves. The experimental dispersion relation was even successfully tested against the theoretical one. Note that the Ginzburg-Landau equation has been widely used in hydrodynamics in order to model thermal convection ${ }^{22}$ or wakes ${ }^{23}$ for instance. Usually, to evaluate the parameters of the equation, experimentalists study the impulse response of the system under its instability threshold. However, when the flow is already in a chaotic regime, the obtention of the parameters is much more delicate and several techniques have already been used more or less successfully: optimization techniques ${ }^{24,25}$ or proper or- thogonal decompositions. ${ }^{26}$ The inversion problem is known to be difficult in this case of chaotic dynamics because of the presence of unavoidable experimental noise that pollutes the data.

\section{DEFINITION OF THE VOLTERRA MODEL}

Since our objective is to quantify the wavefield properties for various Reynolds numbers, it is essential that we use a model which can be identified in an unambiguous way from experimental data, while giving direct access to the physical properties of interest. Volterra models are a good candidate for this. They can accommodate more types of nonlinearities than the Ginzburg-Landau equation, while still offering a straightforward physical interpretation. Our decision to apply such models is further motivated by a desire to leave room for possible unsuspected features that could not be described by the GL equation.

Let $v(x, t, \mathrm{Re})$ be the azimuthal fluid velocity recorded at time $t$, position $x$ and Reynolds number Re. A general dynamical model for the wavefield amplitude is

$$
\frac{\partial v(x, t, \mathrm{Re})}{\partial x}=\mathcal{F}(v(x, t, \mathrm{Re}))
$$

where $\mathcal{F}$ is a continuous, nonlinear and time-invariant operator. We write $\mathcal{F}$ as a Volterra series:

$$
\begin{aligned}
\frac{\partial v_{i}(x, \operatorname{Re})}{\partial x}= & \sum_{k=0}^{\infty} g_{k}(\operatorname{Re}) v_{i-k}(x, \operatorname{Re}) \\
& +\sum_{k=0}^{\infty} \sum_{l=0}^{\infty} g_{k, l}(\operatorname{Re}) v_{i-k}(x, \operatorname{Re}) v_{i-l}(x, \operatorname{Re}) \\
& +\sum_{k=0}^{\infty} \sum_{l=0}^{\infty} \sum_{m=0}^{\infty} g_{k, l, m}(\operatorname{Re}) v_{i-k}(x, \operatorname{Re}) \\
& \times v_{i-l}(x, \operatorname{Re}) v_{i-m}(x, \operatorname{Re})+\cdots .
\end{aligned}
$$

The wavefield $v$ is sampled at a constant rate, so a discrete version of the Volterra series is used here, with the notation $v_{i}(x, \mathrm{Re})=v\left(x, t=t_{i}, \mathrm{Re}\right)$. The coefficients $g_{k}, g_{k, l}$ and $g_{k, l, m}$ are, respectively, called first, second and third order Volterra kernels. In the following, the dependence of $v_{i}$ on $x$ and Re and the dependence of the kernels $g$ on Re will sometimes be omitted, to ease notation.

An expression of the type (1) for $\mathcal{F}$, local in space and convolutive in time, is applicable to a large class of causal systems. In our case, causality is guaranteed by the convective nature of the instability.

In a weakly nonlinear system like the rotating disk boundary layer instability, one would expect the lowest order terms of the series to rule the dynamics. This will indeed be the case, allowing us to truncate the Volterra series after cubic terms. Furthermore, since we are dealing with nonlinearly interacting waves, it is appropriate to consider Fourier modes of the wavefield. The discrete Fourier transform in time of Eq. (1) gives 


$$
\begin{aligned}
\frac{\partial \hat{v}(x, \omega)}{\partial x}= & \Gamma_{1}(\omega) \hat{v}(x, \omega) \\
& +\sum_{\omega_{1}+\omega_{2}=\omega} \Gamma_{2}\left(\omega_{1}, \omega_{2}\right) \hat{v}\left(x, \omega_{1}\right) \hat{v}\left(x, \omega_{2}\right) \\
& +\sum_{\omega_{1}+\omega_{2}+\omega_{3}=\omega} \Gamma_{3}\left(\omega_{1}, \omega_{2}, \omega_{3}\right) \\
& \times \hat{v}\left(x, \omega_{1}\right) \hat{v}\left(x, \omega_{2}\right) \hat{v}\left(x, \omega_{3}\right)+\cdots,
\end{aligned}
$$

where $\hat{v}(\omega)$ stands for the Fourier transform of $v_{i}$ at frequency (unless stated otherwise, we shall call frequency what normally should be the angular frequency) $\omega$. The link between the Volterra kernels in Fourier space and their temporal counterparts is established by Ref. 27:

$$
\begin{aligned}
& \Gamma_{1}(\omega)=\sum_{k=0}^{\infty} g_{k} e^{i \omega k}, \\
& \Gamma_{2}\left(\omega_{1}, \omega_{2}\right)=\sum_{k=0}^{\infty} \sum_{l=0}^{\infty} g_{k, l} e^{i\left(\omega_{1} k+\omega_{2} l\right)}, \\
& \Gamma_{3}\left(\omega_{1}, \omega_{2}, \omega_{3}\right)=\sum_{k=0}^{\infty} \sum_{l=0}^{\infty} \sum_{m=0}^{\infty} g_{k, l, m} e^{i\left(\omega_{1} k+\omega_{2} l+\omega_{3} m\right)}, \ldots
\end{aligned}
$$

Equation (2) shows that the quadratic kernel $\Gamma_{2}\left(\omega_{1}, \omega_{2}\right)$ is associated with three-wave interactions that satisfy the resonance condition $\omega_{1}+\omega_{2}=\omega$, whereas the cubic term $\Gamma_{3}\left(\omega_{1}, \omega_{2}, \omega_{3}\right)$ is related to four-wave interactions $\omega_{1}+\omega_{2}$ $+\omega_{3}=\omega$. Typical examples are decay instabilities for the former and modulational instabilities for the latter. Kernels of different orders are thus directly related to specific physical processes. Likewise, the imaginary part of the linear kernel is associated with the wavefield dispersion and its real part is associated to the linear growth rate. Much work on this topic has been done by Zakharov and his co-workers in the framework of Hamiltonian systems. ${ }^{3}$

Another motivation for using a Fourier representation of the Volterra equation is that it gives access to a key quantity for weakly nonlinear wavefields, which is the spectral energy flux. Although the analysis of such spectral energy transfers falls beyond the scope of this article, we nevertheless mention how they are related to the Volterra kernels. Two applications are described in Refs. 4 and 5.

We start by assuming that the weak turbulence approximation holds, which supposes that the number of interacting waves is sufficiently large for phase decoherence to be effective. In this case, the spectral energy density $E$, which describes the spatial variation of the wavefield energy density, can be defined as

$$
E(x, \omega, \operatorname{Re})=\left\langle\hat{v}^{*}(x, \omega, \operatorname{Re}) \hat{v}\left(x, \omega^{\prime}, \operatorname{Re}\right)\right\rangle \delta_{\omega, \omega^{\prime}},
$$

where $*$ denotes complex conjugation, $\delta$ is the Dirac delta function, and brackets denote ensemble averaging (assumed to be equivalent to time-averaging by ergodicity).

Combining Eqs. (2) and (4) gives the kinetic equation ${ }^{28}$

$$
\begin{aligned}
\frac{\partial E(x, \omega)}{\partial x}= & T_{1}(x, \omega)+\sum_{\omega_{1}+\omega_{2}=\omega} T_{2}\left(x, \omega_{1}, \omega_{2}\right) \\
& +\sum_{\omega_{1}+\omega_{2}+\omega_{3}=\omega} T_{3}\left(x, \omega_{1}, \omega_{2}, \omega_{3}\right)+\cdots
\end{aligned}
$$

The expressions for the first three energy transfer functions are

$$
\begin{aligned}
& T_{1}(x, \omega)=2 \operatorname{Re}_{1}(\omega) E(x, \omega), \\
& T_{2}\left(x, \omega_{1}, \omega_{2}\right)=2 \operatorname{Re} \sum_{\omega_{1}+\omega_{2}=\omega}\left\langle\Gamma_{2}\left(\omega_{1}, \omega_{2}\right) \hat{v}\left(x, \omega_{1}\right)\right. \\
& \left.\times \hat{v}\left(x, \omega_{2}\right) \hat{v}^{*}(x, \omega)\right\rangle, \\
& T_{3}\left(x, \omega_{1}, \omega_{2}, \omega_{3}\right)=2 \operatorname{Re} \sum_{\omega_{1}+\omega_{2}+\omega_{3}=\omega}\left\langle\Gamma_{3}\left(\omega_{1}, \omega_{2}, \omega_{3}\right)\right. \\
& \times \hat{v}\left(x, \omega_{1}\right) \hat{v}\left(x, \omega_{2}\right) \\
& \left.\times \hat{v}\left(x, \omega_{3}\right) \hat{v} *(x, \omega)\right\rangle,
\end{aligned}
$$

where $\operatorname{Re} z$ stands for the real part of $z$. These transfer functions tell us how energy at a given frequency $\omega$ is redistributed to other Fourier modes by nonlinear wave-wave interactions; such couplings are a hallmark of weakly nonlinear systems. A negative quadratic energy transfer $T_{2}\left(\omega_{1}, \omega_{2}\right)$, for example, implies an energy loss at $\omega$ which is transferred by three-wave interactions to Fourier modes with frequencies $\omega_{1}$ and $\omega_{2}$ (with $\omega_{1}+\omega_{2}=\omega$ ). Negative transfers typically arise when a linear instability needs to be saturated. All these quantities can be directly estimated from both the timedomain and frequency-domain Volterra series.

A final modification concerns the estimation of the spatial derivative from the two-point measurements. If the probe separation $\Delta x$ is sufficiently small compared to the wavelength (i.e., if $\mathbf{k} \cdot \Delta \mathbf{x} \ll 1$ ), then we may write

$$
\frac{\partial v_{i}(x, \mathrm{Re})}{\partial x} \approx \frac{v_{i}(x+\Delta x, \mathrm{Re})-v_{i}(x, \mathrm{Re})}{\Delta x} .
$$

Using this result, we may express the Volterra model into the more convenient framework of transfer functions. For this we consider the rotating fluid between the two probes as a causal and open loop system that reacts to a given excitation (= the input, or upstream probe) by giving a response $(=$ the output, or downstream probe). The Volterra model then describes the nonlinear transfer function between the observation points. ${ }^{4}$ It can be written as

$$
\begin{aligned}
u_{i}= & v_{i}(x, \mathrm{Re}) \text { (the input), } \\
y_{i}= & v_{i}(x+\Delta x, \operatorname{Re}) \text { (the output) } \\
= & \sum_{k=0}^{n} \bar{g}_{k} u_{i-k}+\sum_{k=0}^{n} \sum_{l=0}^{n} \bar{g}_{k, l} u_{i-k} u_{i-l} \\
& +\sum_{k=0}^{n} \sum_{l=0}^{n} \sum_{m=0}^{n} \bar{g}_{k, l, m} u_{i-k} u_{i-l} u_{i-m}+\cdots+\epsilon_{i},
\end{aligned}
$$


where $\epsilon_{i}$ is the residual error to be minimized. The coefficients $\bar{g}$ of the transfer function are related to the Volterra kernels [Eq. (1)] by

$$
g=\frac{\bar{g}}{\Delta x},
$$

except for the linear term of zero lag, $g_{0}=\left(\bar{g}_{0}-1\right) / \Delta x$. Note that in contrast to Eq. (1), the number of considered lags is now bounded by $n$, to comply with the finite length of the time series.

Although the closeness of the probes (i.e., the condition $\mathbf{k} \cdot \Delta \mathbf{x} \ll 1$ ) was imposed above to approximate the spatial derivative of the wavefield, it can be relaxed in situations like here where we are dealing with an amplitude equation and one dominating Fourier mode. What matters is that the characteristic scale associated with the evolution of the wave envelope and not the carrier itself. This scale should be small as compared to the probe spacing, and yet large enough to make nonlinear effects easily distinguishable. In our experiment $\mathbf{k} \cdot \Delta \mathbf{x} \approx \mathbf{1}$ provides a reasonable compromise in the linear and weakly nonlinear regimes.

\section{VOLTERRA MODEL IDENTIFICATION}

Now that we have selected a physically relevant model, the next step consists in identifying that model from finite and noise-corrupted time series. There is vast literature on theoretical properties of Volterra models ${ }^{2,29,30}$ but relatively little is known about their statistical properties and their inference from experimental data.

Most approaches to Volterra model identification so far have been carried out in the frequency domain. ${ }^{4,5,31,32}$ This is indeed justified when the system dynamics is dominated by a limited number of interacting modes. The advantage of a model equation like (2) is that the unknown kernels enter the problem in a linear way, which means that they can be estimated by standard linear regression techniques.

Unfortunately, frequency-domain identification leads to ill-conditioned problems. Because of this, most studies have been restricted so far to quadratically nonlinear models only, with relatively few Fourier modes. It is therefore of prime importance to find a parsimonious model if we wish to investigate cubic and higher order wave interactions in our experiment.

To alleviate the problem of ill-conditioning, we estimate the Volterra model in the time domain, and subsequently compute the kernels in the Fourier domain using Eq. (3). Indeed, the significant kernels are generally easier to identify and to validate in the time domain. Quite often, less than a dozen suitably chosen kernels suffice to fit the data with reasonable accuracy.

It should be noted that the time-domain Volterra model (1) belongs to the class of more general parametric models called Nonlinear AutoRegressive Moving Average with eXogeneous inputs (NARMAX). The ability of such models to fit various types of nonlinear systems has led to a large interest in them. ${ }^{30,33,34}$ In NARMAX models, the output $y_{i}$ at a given time is given by

$$
\begin{aligned}
y_{i}= & \mathcal{F}\left[y_{i-1}, \ldots, y_{i-n}, u_{i}, u_{i-1}, \ldots, u_{i-n},\right. \\
& \left.\epsilon_{i-1}, \ldots, \epsilon_{i-n}\right]+\epsilon_{i},
\end{aligned}
$$

where $u_{i}$ is the input, $\epsilon_{i}$ the residual error and $\mathcal{F}$ a polynomial. In contrast to the Volterra series, the output of such models depends on combinations involving past outputs, past and present inputs and past residual errors. This enhances the fitting capacity of the NARMAX model considerably.

Physical considerations suggest that our data should be better described by NARMAX than by Volterra models. Indeed, the former can handle various features (resonances, long time constants) which Volterra models cannot. However, it is also known that NARMAX models can be arbitrarily well approximated by a Volterra series, at the expense of a larger (or possibly infinite) number of parameters.

Our main incentive for choosing Volterra instead of NARMAX models, is due to the smaller computational investment of the former. Furthermore, some of the theoretical results needed to recover frequency-domain expressions from NARMAX models aren't yet in the public domain. Our work should in this sense be considered as a first step toward a better adapted model.

We are now left with the determination of the significant terms in Eq. (5). This is the problem of model structure selection, which is crucial for a successful identification. Parsimony is necessary to avoid ill-conditioning, but the selection of the significant kernels is equally important for a proper modeling.

In practice, one starts with a model that has large lags $n$ and a relatively high polynomial degree. Then, by successive elimination, the unnecessary kernels are discarded until a "minimum model"' is retained. The Error Reduction Ratio (ERR) criterion is generally used for that purpose. ${ }^{35,36}$ Its definition, together with practical aspects of nonlinear model identification, are deferred to the Appendix.

The final step is the estimation and the validation of the model parameters. There are few rigorous results on nonlinear model validation; to a large extent one has to rely on better understood results from the linear case; see Ref. 37. Among the quantities to be studied are the confidence intervals of the parameters, the dependence of the results on the model choice and a statistical analysis of the residuals $\epsilon$. Visual inspection of the latter should not reveal any correlation with the in- or output. This can be further quantified by hypotheses testing the level of cross correlation between $\epsilon$, and $y$ or $u$.

\section{APPLICATION TO THE ROTATING DISK FLOW}

The present analysis is based on two-point measurements carried out with a fixed probe separation of $\Delta x=8$ $\mathrm{mm}$, at a constant disk rotation speed. We consider 18 series of 32768 samples each, representing 64 rotation periods each. These series correspond to Reynolds numbers going from 250 to 505 , which roughly encompasses the transition from laminar to developed turbulent motion.

Two problems we had to overcome before analyzing the data were the oblique orientation of the probes (see Fig. 2) and the lack of calibration. The former means that the two 
probes do not observe exactly the same structures. For scales of the order of the probe separation, however, the waves are essentially planar and so this problem can be neglected.

Calibration of the probes is compulsory for an estimation of the linear growth rate. To overcome its absence, we used ad hoc methods to correct the signal levels. Satisfactory results were obtained by equaling the wavefield power spectral density in a limited band around the frequency $\omega_{c}$ of the fundamental. This choice is justified by the saturation of the fundamental mode, which should not vary much over the range of the probe separation. Adaptive high pass filtering was also applied in order to eliminate low frequency disturbances observed at low Reynolds numbers.

The Volterra model identification is now detailed for a particular data set, which corresponds to $\mathrm{Re}=387$. First, the most significant kernels are identified, using the procedure described in Sec. IV. For this particular data set, Akaike's information criterion (see the Appendix) suggests that a model with seven terms only represents a satisfactory tradeoff between parsimony and the ability to fit the results. Out of these seven terms, two are linear and five are cubic. A least squares fit yields the model coefficients:

$$
\begin{aligned}
y_{i}= & -0.7610 u_{i-8}+0.0032 u_{i-8} u_{i-1}^{2}-0.0105 u_{i-24}^{2} u_{i-3} \\
& +0.1022 u_{i}+0.0017 u_{i-23} u_{i-20} u_{i-8} \\
& -0.0115 u_{i-14} u_{i-3} u_{i-1}+0.0103 u_{i-23} u_{i-11} u_{i}+\epsilon_{i} .
\end{aligned}
$$

The uncertainty level of these coefficients is typically less than $10 \%$ but increases with the model order. The different terms are arranged here in decreasing Error Reduction Ratio (ERR) order, that is, the leading terms are the most important ones for predicting the output. One should not pay too much attention to the values of the delays, especially those appearing in the nonlinear terms, since what eventually matters are the combinations of the terms, as given in Eq. (3).

The most significant kernels are chosen among all possible combinations of linear, quadratic and cubic terms, with a memory (i.e., a number of delays $n$ ) equaling up to three wavefield periods. We also ran the procedure with models including fourth and fifth order terms, in order to test for the possible existence of higher order nonlinearities. All these tests were negative, suggesting that no higher order terms are needed to properly model the wavefield dynamics. This is an important point, since it justifies the truncation of the Volterra series at cubic terms. Such a truncation not only eases the identification procedure, but also provides a convenient closure for the analytical expressions. This closure is often imposed a priori as a working hypothesis while we deduce it from the flow.

Another noticeable result is the strong ordering of the Volterra kernels, regardless of the value of the Reynolds number. Linear terms always prevail, as it is expected for this type of experiment. The dominant nonlinear term, however, is not quadratic but cubic. Indeed, quadratic terms always show up much later in the sequence, thereby attesting the relative weakness of three-wave interactions as compared to four-wave interactions. The same type of ordering is found in the nonlinear interaction of gravity waves; both have their origin rooted in the nonlinear dispersion relation of the wavefield, which forbids interactions between waves satisfying the resonance condition $\omega\left(\mathbf{k}_{1}+\mathbf{k}_{2}\right)=\omega\left(\mathbf{k}_{1}\right)$ $+\omega\left(\mathbf{k}_{2}\right)$, whereas four-wave interactions of the type $\omega\left(\mathbf{k}_{1}\right.$ $\left.+\mathbf{k}_{2}+\mathbf{k}_{3}\right)=\omega\left(\mathbf{k}_{1}\right)+\omega\left(\mathbf{k}_{2}\right)+\omega\left(\mathbf{k}_{3}\right)$ are compatible with the dispersion relation.

One of the common signatures of three-wave interactions is an up-down asymmetry in the wavefield, which results in a generation of harmonics. A higher order spectral analysis of the wavefield confirms the existence of weak three-wave interactions, and the power spectral density indeed has a small peak at $2 \omega_{c}$. These interactions are strongest at intermediate Reynolds numbers $(330<\operatorname{Re}<440)$, for which the nonlinearity is already significant while the dispersion relation still allows for some three-wave interaction.

Four-wave interactions can have various signatures. We are dealing here with a coupling between frequencies located in narrow bands that are centered on the fundamental frequency. These different results are illustrated in Fig. 4 which compares the in- and output, the model prediction and the linear and nonlinear constituents of the latter. A model with 11 terms was used in order to include the leading quadratic term.

As far as the model performance is concerned, Fig. 4(b) shows that a low order Volterra model succeeds relatively well in reproducing the real data. The fit can certainly be improved since the residuals are neither white nor uncorrelated with the input. NARMAX models may be more appropriate here, but the relatively low level of residual noise gives us confidence that the Volterra model still captures the salient features of the wavefield dynamics. The general performance gradually degrades as the wavefield becomes turbulent, essentially because the probe spacing is too large as compared to the characteristic scale over which the wavefield evolves. More closely spaced probes would certainly have given better results here.

The decomposition of the Volterra model output into its different linear and nonlinear constituents is an interesting exercise, since it reveals how each type of nonlinearity contributes to the wavefield dynamics. Figures 4(d) $-4(\mathrm{f})$ confirm the ordering (linear $>$ cubic $>$ quadratic) of the kernels. The main effect of the linear term is to fit the convective motion by shifting the wave pattern in time by about 30 (msec). This delay is equivalent to one period of the fundamental mode. Notice that a linear prediction fails to reproduce the phase shift observed at the center of the pulse, for which a nonlinear correction is needed. This 40 to 50 degree phase shift is taken into account by the cubic term, whose effect therefore amounts to an amplitude dependence in the dispersion relation.

We must stress that these results are systematic; by including more or less terms in the Volterra series one changes the kernels in Fourier space a little, but the conclusions remain unaffected. We now consider how Volterra kernels are related to the parameters of the GL equation.

\section{RELATION TO THE COMPLEX GINZBURG-LANDAU EQUATION}

The (one-dimensional) Ginzburg-Landau equation describes the evolution, near the critical linear threshold $\mathrm{Re}_{c}$, 

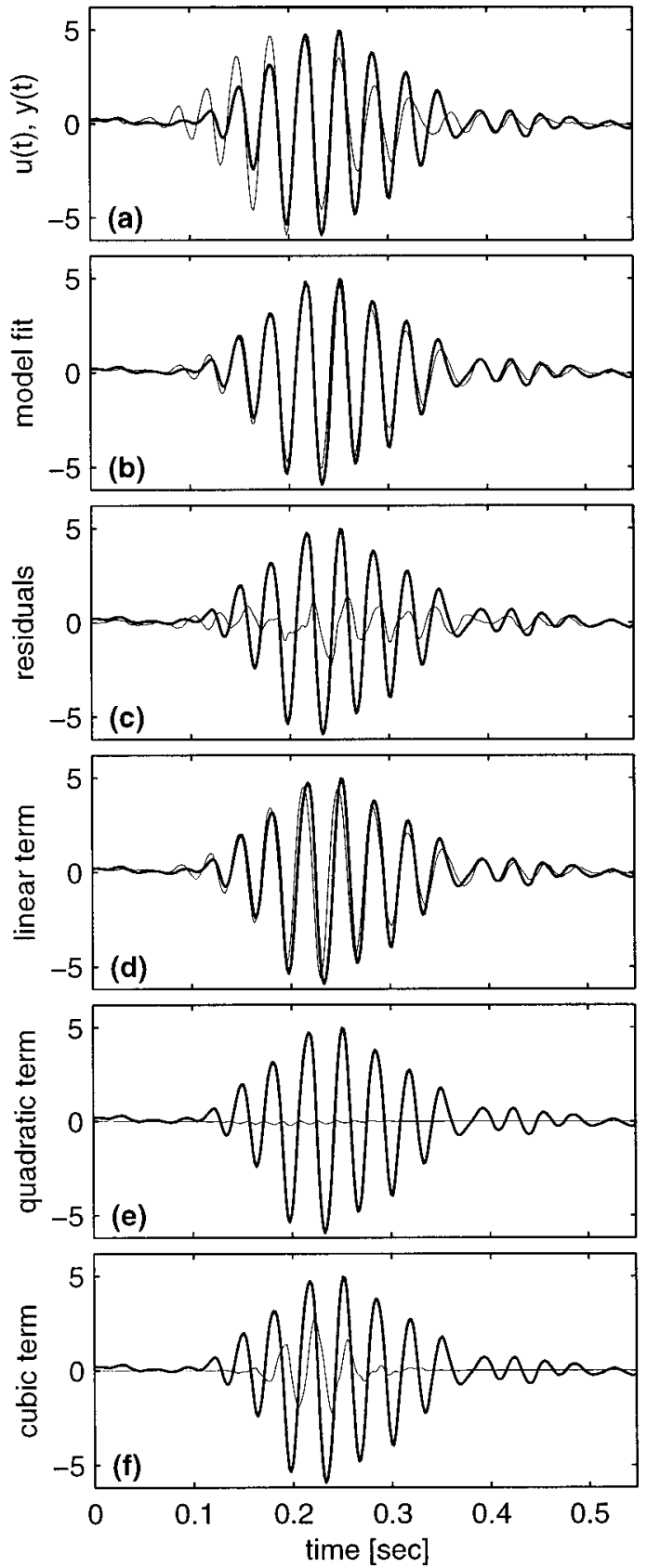

FIG. 4. Excerpt of the wavefield amplitude at $R e=387$ showing from top to bottom (with the downstream probe always in bold): (a) the measured inand output, (b) the measured output and its prediction, (c) the measured output and the residuals, (d) the measured output and the linear constituent of the prediction, (e) the measured output and the quadratic constituent of the prediction, and (f) the measured output and the cubic constituent of the prediction. The measured signals are centered and reduced.

of a linearly unstable wave packet centered on the marginal wave of frequency $\omega_{c}$, wavenumber $k_{c}$, and propagating in the direction $x$. The hydrodynamic field defining the wave packet, $v(x, t)$, can be written as

$$
v(x, t)=A(x, t) e^{i k_{c} x-i \omega_{c} t}+\text { c.c. },
$$

where $A(x, t)$ is a complex function, slowly varying in space and time. The GL equation is an equation for the amplitude $A(x, t)$, that, taking into account the lowest order nonlinearity, has the form

$$
\begin{aligned}
\tau_{0}\left[\frac{\partial A(x, t)}{\partial t}+V_{g} \frac{\partial A(x, t)}{\partial x}\right]= & \mu A+\xi_{0}^{2}\left(1+i c_{1}\right) \frac{\partial^{2} A(x, t)}{\partial x^{2}} \\
& -g\left(1+i c_{2}\right)|A(x, t)|^{2} A(x, t),
\end{aligned}
$$

where

$$
\mu=\frac{\operatorname{Re}-\operatorname{Re}_{c}}{\operatorname{Re}_{c}},
$$

$V_{g}$ is the group velocity, $\tau_{0}$ and $\xi_{0}$ are the characteristic time and length of the instability, $c_{1}$ and $c_{2}$ are nondimensional and $g$ is the saturation parameter.

The general solution of a linear stability hydrodynamic problem can be expressed using a frequency and a wavenumber that verify a complex dispersion relation $\omega=\omega(k, \mathrm{Re})$. The coefficients $\tau_{0}, V_{g}, \xi_{0}, c_{1}$ are related to the Taylor expansion of the frequency $\omega(k, \mathrm{Re})$ near the critical threshold in the following way: ${ }^{9}$

$$
\begin{aligned}
& \tau_{0}^{-1}=-\left.i \operatorname{Re}_{c} \frac{\partial \omega}{\partial \operatorname{Re}}\right|_{c}, \\
& V_{g}=\left.\frac{\partial \omega}{\partial k}\right|_{c}, \\
& \xi_{0}^{2}\left(1+i c_{1}\right)=\left.\frac{i \tau_{0}}{2} \frac{\partial^{2} \omega}{\partial k^{2}}\right|_{c},
\end{aligned}
$$

where $\left.\right|_{c}$ means that the partial derivatives are calculated at the critical point $\operatorname{Re}=\operatorname{Re}_{c}, k=k_{c}$.

If the solution $A(x, t)$ is developed in a temporal Fourier series,

$$
A(x, t)=\sum_{\nu} \hat{A}(x, \nu) e^{-i \nu t},
$$

it is easy to verify that Eq. (8) is equivalent to

$$
\begin{aligned}
0= & i \nu \hat{A}(x, \nu)-\left.\frac{\partial \omega}{\partial k}\right|_{c} \frac{\partial \hat{A}(x, \nu)}{\partial x}+\left.\frac{i}{2} \frac{\partial^{2} \omega}{\partial k^{2}}\right|_{c} \\
& \times \frac{\partial^{2} \hat{A}(x, \nu)}{\partial x^{2}}-\left.i \frac{\partial \omega}{\partial \operatorname{Re}}\right|_{c}\left(\operatorname{Re}-\operatorname{Re}_{c}\right) \hat{A}(x, \nu) \\
& -q \sum_{\nu_{1}+\nu_{2}+\nu_{3}=\nu} \hat{A}\left(x, \nu_{1}\right) \hat{A}\left(x, \nu_{2}\right) \hat{A}^{*}\left(x,-\nu_{3}\right),
\end{aligned}
$$

where $q$ is defined by

$$
q=\frac{g\left(1+i c_{2}\right)}{\tau_{0}} .
$$

This one-dimensional equation can describe, at least approximately, our 3-dimensional system, because the vortices produced by the cross-flow instability have a definite propagation direction, so that the system is essentially convective. This one-dimensional approach has been successfully applied in Ref. 7.

Now compare (12) to the Volterra Eq. (2): 


$$
\begin{aligned}
\frac{\partial \hat{v}(x, \omega)}{\partial x}= & \Gamma_{1}(\omega) \hat{v}(x, \omega) \\
& +\sum_{\omega_{1}+\omega_{2}=\omega} \Gamma_{2}\left(\omega_{1}, \omega_{2}\right) \hat{v}\left(x, \omega_{1}\right) \hat{v}\left(x, \omega_{2}\right) \\
& +\sum_{\omega_{1}+\omega_{2}+\omega_{3}=\omega} \Gamma_{3}\left(\omega_{1}, \omega_{2}, \omega_{3}\right) \hat{v}\left(x, \omega_{1}\right) \\
& \times \hat{v}\left(x, \omega_{2}\right) \hat{v}\left(x, \omega_{3}\right),
\end{aligned}
$$

considered at $\operatorname{Re} \simeq \operatorname{Re}_{c}, \omega \simeq \omega_{c}$, and the coordinate $x$ is measured along the propagation direction. Both equations describe the same physical situation. There must then be a relation between the coefficients of the GL equation and the kernels $\Gamma_{i}$, that we will now determine.

One remark is in order: the Volterra expansion has a structure that is very different from that of the GL equation. The GL equation involves by construction the slowly varying amplitude of the fundamental wave $\left(k_{c}, \omega_{c}\right), A(x, t)$, whose Fourier components $\hat{A}(x, \nu)$ are non negligible only for $\nu$ small. On the other hand, the lowest order nonlinear term in the Volterra expansion (14), considered at $\mathrm{Re}$ $\simeq \operatorname{Re}_{c}, \omega \simeq \omega_{c}$, is equal to zero if, in the sums, only the frequencies close to the fundamental one $\omega_{c}$ are taken into account, so that we are obliged to include at least the first harmonic of the marginal wave in our description. In fact, the sum $\sum_{\omega_{1}+\omega_{2}=\omega \simeq \omega_{c}}$ takes its lowest order contribution from the terms $\omega_{1} \simeq 2 \omega_{c}, \omega_{2} \simeq-\omega_{c}$, and $\omega_{1} \simeq-\omega_{c}, \omega_{2}$ $\simeq 2 \omega_{c}$.
More precisely, we will suppose that, near the critical threshold $\operatorname{Re}=\operatorname{Re}_{c}, v(x, t)$ has the following form:

$$
v(x, t)=\left[A(x, t) e^{i k_{c} x-i \omega_{c} t}+B(x, t) e^{i 2 k_{c} x-i 2 \omega_{c} t}\right]+\text { c.c. },
$$

with $A(x, t), B(x, t)$ slowly varying in space and time. The GL equation (14) is then an equation for $A(x, t)$ only, whereas the quadratic term in (8) at $\omega \simeq \omega_{c}$ will contain $B(x, t)$ too.

Equation (15) gives in Fourier space,

$$
\begin{aligned}
\hat{v}(x, \omega)= & e^{i k_{c} x} \hat{A}\left(x, \omega-\omega_{c}\right) \\
& +e^{-i k_{c} x} \hat{A}^{*}\left(x,-\omega-\omega_{c}\right)+e^{i 2 k_{c} x} \hat{B}\left(x, \omega-2 \omega_{c}\right) \\
& +e^{-i 2 k_{c} x} \hat{B}^{*}\left(x,-\omega-2 \omega_{c}\right) .
\end{aligned}
$$

The fact that $A(x, t), B(x, t)$ are slowly varying means that their Fourier components $\hat{A}(x, \nu), \hat{B}(x, \nu)$ are non negligible only for $\nu \simeq 0$. This implies, of course,

$$
\begin{aligned}
& \hat{v}\left(x, \omega \simeq \omega_{c}\right)=e^{i k_{c} x} \hat{A}\left(x, \omega-\omega_{c}\right), \\
& \hat{v}\left(x, \omega \simeq 2 \omega_{c}\right)=e^{i 2 k_{c} x} \hat{B}\left(x, \omega-2 \omega_{c}\right), \\
& \hat{v}\left(x, \omega \simeq-\omega_{c}\right)=e^{-i k_{c} x} \hat{A}^{*}\left(x,-\omega-\omega_{c}\right), \\
& \hat{v}\left(x, \omega \simeq-2 \omega_{c}\right)=e^{-i 2 k_{c} x} \hat{B}^{*}\left(x,-\omega-2 \omega_{c}\right) .
\end{aligned}
$$

Equation (14) gives then, for $\omega$ close to $\omega_{c}$,

$$
\begin{aligned}
\frac{\partial \hat{A}\left(x, \omega-\omega_{c}\right)}{\partial x}= & {\left[\Gamma_{1}(\omega)-i k_{c}\right] \hat{A}\left(x, \omega-\omega_{c}\right)+2 \sum_{\substack{\omega_{1}+\omega_{2}=\omega, \omega_{1} \simeq 2 \omega_{c}, \omega_{2} \simeq-\omega_{c}}} \Gamma_{2}\left(\omega_{1}, \omega_{2}\right) \hat{B}\left(x, \omega_{1}-2 \omega_{c}\right) \hat{A}^{*}\left(x,-\omega_{2}-\omega_{c}\right) } \\
& +3 \sum_{\substack{\omega_{1}+\omega_{2}+\omega_{3}=\omega, \omega_{1} \simeq \omega_{2} \simeq-\omega_{3} \simeq \omega_{c}}} \Gamma_{3}\left(\omega_{1}, \omega_{2}, \omega_{3}\right) \hat{A}\left(x, \omega_{1}-\omega_{c}\right) \hat{A}\left(x, \omega_{2}-\omega_{c}\right) \hat{A} *\left(x,-\omega_{3}-\omega_{c}\right),
\end{aligned}
$$

where we used the symmetry of the kernels $\Gamma_{i}$ with respect to their $i$ arguments.

We want an equation involving only $\hat{A}(x, \nu)$, in order to establish a relation with the GL equation. We will then consider the second order Volterra expansion (14) for $\omega_{1}$ $\simeq 2 \omega_{c}$ :

$$
\begin{aligned}
\frac{\partial \hat{B}\left(x, \omega_{1}-2 \omega_{c}\right)}{\partial x}= & {\left[\Gamma_{1}\left(\omega_{1}\right)-2 i k_{c}\right] \hat{B}\left(x, \omega_{1}-2 \omega_{c}\right) } \\
& +\sum_{\substack{\omega_{3}+\omega_{4}=\omega_{1}, \omega_{3} \simeq \omega_{4} \simeq \omega_{c}}} \Gamma_{2}\left(\omega_{3}, \omega_{4}\right) \\
& \times \hat{A}\left(x, \omega_{3}-\omega_{c}\right) \hat{A}\left(x, \omega_{4}-\omega_{c}\right) .
\end{aligned}
$$

Near the critical point threshold, the real part of $\hat{\Gamma}_{1}\left(2 \omega_{c}\right)$ (the spatial growth rate of the first harmonic of the marginal wave) is large and negative. The adiabatic approximation is thus applicable, ${ }^{38}$ so that

$$
\begin{aligned}
\hat{B}\left(x, \omega_{1}-2 \omega_{c}\right) \simeq & -\frac{1}{\operatorname{Re}\left(\Gamma_{1}\left(\omega_{1}\right)\right)} \\
& \times \sum_{\substack{\omega_{3}+\omega_{4}=\omega_{1}, \omega_{3} \simeq \omega_{4} \simeq \omega_{c}}} \Gamma_{2}\left(\omega_{3}, \omega_{4}\right) \\
& \times \hat{A}\left(x, \omega_{3}-\omega_{c}\right) \hat{A}\left(x, \omega_{4}-\omega_{c}\right) .
\end{aligned}
$$

Substituting this expression in (16), we get 


$$
\begin{aligned}
\frac{\partial \hat{A}\left(x, \omega-\omega_{c}\right)}{\partial x}= & {\left[\Gamma_{1}(\omega)-i k_{c}\right] \hat{A}\left(x, \omega-\omega_{c}\right)-2 \sum_{\substack{\omega_{1}+\omega_{2}=\omega, \omega_{1} \simeq 2 \omega_{c}, \omega_{2} \simeq-\omega_{c}}} \sum_{\substack{\omega_{3}+\omega_{4}=\omega_{1}, \omega_{3} \simeq \omega_{4} \simeq \omega_{c}}} \frac{\Gamma_{2}\left(\omega_{1}, \omega_{2}\right) \Gamma_{2}\left(\omega_{3}, \omega_{4}\right)}{\operatorname{Re}\left(\Gamma_{1}\left(\omega_{1}\right)\right)} \hat{A}\left(x, \omega_{3}-\omega_{c}\right) \hat{A}\left(x, \omega_{4}-\omega_{c}\right) } \\
& \times \hat{A}^{*}\left(x,-\omega_{2}-\omega_{c}\right)+3 \sum_{\substack{\omega_{1}+\omega_{2}+\omega_{3}=\omega_{1} \\
\omega_{1} \simeq \omega_{2} \simeq-\omega_{3} \simeq \omega_{c}}} \Gamma_{3}\left(\omega_{1}, \omega_{2}, \omega_{3}\right) \hat{A}\left(x, \omega_{1}-\omega_{c}\right) \hat{A}\left(x, \omega_{2}-\omega_{c}\right) \hat{A} *\left(x,-\omega_{3}-\omega_{c}\right),
\end{aligned}
$$

which can be rewritten as

$$
\begin{aligned}
\frac{\partial \hat{A}\left(x, \omega-\omega_{c}\right)}{\partial x}= & {\left[\Gamma_{1}(\omega)-i k_{c}\right] \hat{A}\left(x, \omega-\omega_{c}\right) } \\
& +\sum_{\substack{\omega_{1}+\omega_{2}+\omega_{3}=\omega, \omega_{1} \simeq \omega_{2} \simeq-\omega_{3} \simeq \omega_{c}}} \Lambda\left(\omega_{1}, \omega_{2}, \omega_{3}\right) \\
& \times \hat{A}\left(x, \omega_{1}-\omega_{c}\right) \hat{A}\left(x, \omega_{2}-\omega_{c}\right) \\
& \times \hat{A}^{*}\left(x,-\omega_{3}-\omega_{c}\right),
\end{aligned}
$$

where $\Lambda$ is defined by

$$
\begin{aligned}
\Lambda\left(\omega_{1}, \omega_{2}, \omega_{3}\right)= & -2 \frac{\hat{\Gamma}_{2}\left(\omega_{1}, \omega_{2}\right) \hat{\Gamma}_{2}\left(\omega-\omega_{3}, \omega_{3}\right)}{\operatorname{Re}\left(\hat{\Gamma}_{1}\left(\omega-\omega_{3}\right)\right)} \\
& +3 \hat{\Gamma}_{3}\left(\omega_{1}, \omega_{2}, \omega_{3}\right) .
\end{aligned}
$$

Notice that in the cubic part of Eq. (16), we did not take into account the possible terms proportional to $\hat{A} \hat{B} \hat{B}^{*}$; this is justified a posteriori by the fact that, to leading order, $\hat{B}$ $\propto \hat{A}^{2}$, and we are considering only the cubic nonlinear term in the GL equation.
Differentiating Eq. (19) with respect to $x$ we get

$$
\begin{aligned}
\frac{\partial^{2} \hat{A}\left(x, \omega-\omega_{c}\right)}{\partial x^{2}}= & {\left[\Gamma_{1}(\omega)-i k_{c}\right]^{2} \hat{A}\left(x, \omega-\omega_{c}\right) } \\
& +\sum_{\substack{\omega_{1}+\omega_{2}+\omega_{3}=\omega, \omega_{1} \simeq \omega_{2} \simeq-\omega_{3} \simeq \omega_{c}}} \hat{A}\left(x, \omega_{1}-\omega_{c}\right) \\
& \times \hat{A}\left(x, \omega_{2}-\omega_{c}\right) \hat{A}^{*}\left(x,-\omega_{3}-\omega_{c}\right) \\
& \times \Lambda\left(\omega_{1}, \omega_{2}, \omega_{3}\right)\left[\left(\Gamma_{1}(\omega)-i k_{c}\right)\right. \\
& +\left(\Gamma_{1}\left(\omega_{1}\right)-i k_{c}\right)+\left(\Gamma_{1}\left(\omega_{2}\right)-i k_{c}\right) \\
& \left.+\left(\Gamma_{1}\left(\omega_{3}\right)+i k_{c}\right)\right] .
\end{aligned}
$$

Again, we keep only terms proportional to $A^{n}$ with $n$ $\leqslant 3$.

We will now replace the expressions (19) and (21) for $\partial \hat{A}(x, \nu) / \partial x$ and $\partial^{2} \hat{A}(x, \nu) / \partial x^{2}$ in the GL equation (12). We get

$$
\begin{aligned}
0= & i \hat{A}\left(x, \omega-\omega_{c}\right)\left\{\left(\omega-\omega_{c}\right)+\left.i \frac{\partial \omega}{\partial k}\right|_{c}\left[\Gamma_{1}(\omega)-i k_{c}\right]-\left.\frac{\partial \omega}{\partial \operatorname{Re}}\right|_{c}\left(\operatorname{Re}-\operatorname{Re}_{c}\right)+\left.\frac{1}{2} \frac{\partial^{2} \omega}{\partial k^{2}}\right|_{c}\left[\Gamma_{1}(\omega)-i k_{c}\right]^{2}\right\} \\
& +\sum_{\substack{\omega_{1}+\omega_{2}+\omega_{3}=\omega, \omega_{1} \simeq \omega_{2} \simeq-\omega_{3} \simeq \omega_{c}}} \hat{A}\left(x, \omega_{1}-\omega_{c}\right) \hat{A}\left(x, \omega_{2}-\omega_{c}\right) \hat{A}^{*}\left(x,-\omega_{3}-\omega_{c}\right)\left\{-\left.\frac{\partial \omega}{\partial k}\right|_{c} \Lambda\left(\omega_{1}, \omega_{2}, \omega_{3}\right)\right. \\
& \left.\left.+\left.\frac{i}{2} \frac{\partial^{2} \omega}{\partial k^{2}}\right|_{c} \Lambda\left(\omega_{1}, \omega_{2}, \omega_{3}\right)\left[\Gamma_{1}(\omega)+\Gamma_{1}\left(\omega_{1}\right)+\Gamma_{1}\left(\omega_{2}\right)+\Gamma_{1}\left(\omega_{3}\right)-2 i k_{c}\right)\right]-q\right\} .
\end{aligned}
$$

The first thing to observe is that the expression multiplying $\hat{A}\left(x, \omega-\omega_{c}\right)$ on the first line is approximately zero, for $\operatorname{Re}$ close to $\operatorname{Re}_{c}$ and $\omega$ close to $\omega_{c}$. In fact, the first Volterra kernel $\Gamma_{1}$ is obviously related to the inverse of the complex dispersion relation $\omega(k, \mathrm{Re})$ :

$$
\Gamma_{1}(\omega, \operatorname{Re})=i k(\omega, \operatorname{Re}) .
$$

Near the critical point we have

$$
\begin{aligned}
k(\omega, \operatorname{Re}) \simeq & k_{c}+\left.\frac{\partial k}{\partial \omega}\right|_{c}\left(\omega-\omega_{c}\right)+\left.\frac{\partial k}{\partial \operatorname{Re}}\right|_{c}\left(\operatorname{Re}-\operatorname{Re}_{c}\right) \\
& +\left.\frac{1}{2} \frac{\partial^{2} k}{\partial \omega^{2}}\right|_{c}\left(\omega-\omega_{c}\right)^{2} .
\end{aligned}
$$

Replacing this expression for $\Gamma_{1}(\omega, \operatorname{Re})$ in $(22)$, we see that the term linear in $\hat{A}\left(x, \omega-\omega_{c}\right)$ is zero, thanks to the following relations: 


$$
\begin{aligned}
& \left.\left.\frac{\partial k}{\partial \omega}\right|_{c} \frac{\partial \omega}{\partial k}\right|_{c}=1, \\
& \left.\left.\frac{\partial^{2} k}{\partial \omega^{2}}\right|_{c} \frac{\partial \omega}{\partial k}\right|_{c}+\left.\left(\left.\frac{\partial k}{\partial \omega}\right|_{c}\right) \frac{\partial^{2} \omega}{\partial k^{2}}\right|_{c}=0, \\
& \left.\frac{\partial \omega}{\partial \operatorname{Re}}\right|_{c}+\left.\left.\frac{\partial \omega}{\partial k}\right|_{c} \frac{\partial k}{\partial \operatorname{Re}}\right|_{c} \simeq 0 .
\end{aligned}
$$

Equations (24) and (25) are general, and express the relation existing between the first and second derivatives of a function of complex variable and the derivatives of the inverse function. Equation (26) is specific to a dipersion relation, and is proved in Ref. 39.

Let us now consider the cubic term in Eq. (22):

$$
\begin{aligned}
& \sum_{\substack{\omega_{1}+\omega_{2}+\omega_{3}=\omega, \omega_{1} \simeq \omega_{2} \simeq-\omega_{3} \simeq \omega_{c}}} \hat{A}\left(x, \omega_{1}-\omega_{c}\right) \hat{A}\left(x, \omega_{2}-\omega_{c}\right) \hat{A} *\left(x,-\omega_{3}-\omega_{c}\right) \\
& \times\left\{-\left.\frac{\partial \omega}{\partial k}\right|_{c} \Lambda\left(\omega_{1}, \omega_{2}, \omega_{3}\right)+\left.\frac{i}{2} \frac{\partial^{2} \omega}{\partial k^{2}}\right|_{c} \Lambda\left(\omega_{1}, \omega_{2}, \omega_{3}\right)\right. \\
& \left.\left.\times\left[\Gamma_{1}(\omega)+\Gamma_{1}\left(\omega_{1}\right)+\Gamma_{1}\left(\omega_{2}\right)+\Gamma_{1}\left(\omega_{3}\right)-2 i k_{c}\right)\right]-q\right\} \\
& \equiv \sum_{\substack{\omega_{1}+\omega_{2}+\omega_{3}=\omega_{1} \\
\omega_{1} \simeq \omega_{2} \simeq-\omega_{3} \simeq \omega_{c}}} \hat{A}\left(x, \omega_{1}-\omega_{c}\right) \hat{A}\left(x, \omega_{2}-\omega_{c}\right) \\
& \quad \times \hat{A}^{*}\left(x,-\omega_{3}-\omega_{c}\right)\left[Q\left(\omega_{1}, \omega_{2}, \omega_{3}\right)-q\right] .
\end{aligned}
$$

Expanding $Q\left(\omega_{1}, \omega_{2}, \omega_{3}\right)$ around the critical point $\left(\omega_{c}, \omega_{c},-\omega_{c}\right)$, like it was done for $\Gamma_{1}(\omega)$, we see that the parameter $q$ has to be identified with the leading term $Q\left(\omega_{c}, \omega_{c},-\omega_{c}\right)$. Since $\Gamma_{1}\left(\omega_{c}\right)=i k_{c}$, we get

$$
\begin{aligned}
q= & -\left.\frac{\partial \omega}{\partial k}\right|_{c} \Lambda\left(\omega_{c}, \omega_{c}, \omega_{c}\right) \\
= & \left.\frac{\partial \omega}{\partial k}\right|_{c}\left[2 \frac{\Gamma_{2}\left(\omega_{c}, \omega_{c}\right) \Gamma_{2}\left(2 \omega_{c},-\omega_{c}\right)}{\operatorname{Re}\left(\Gamma_{1}\left(2 \omega_{c}\right)\right)}\right. \\
& \left.-3 \Gamma_{3}\left(\omega_{c}, \omega_{c},-\omega_{c}\right)\right] .
\end{aligned}
$$

Explicitly, using Eqs. (9)-(11), (23), (24)-(26) we get

$$
\begin{aligned}
& V_{g}=i\left[\left.\frac{\partial \hat{\Gamma}_{1}}{\partial \omega}\right|_{c}\right]^{-1}, \\
& \tau_{0}=\left[\left.\operatorname{Re}_{c} V_{g} \frac{\partial \hat{\Gamma}_{1}}{\partial \operatorname{Re}}\right|_{c} ^{-1},\right.
\end{aligned}
$$

$$
\begin{aligned}
\xi_{0}^{2}\left(1+i c_{1}\right) & =-\left.\frac{\tau_{0}}{2} V_{g}^{3} \frac{\partial^{2} \hat{\Gamma}_{1}}{\partial \omega^{2}}\right|_{c}, \\
g\left(1+i c_{2}\right)= & V_{g} \tau_{0}\left[2 \frac{\Gamma_{2}\left(\omega_{c}, \omega_{c}\right) \Gamma_{2}\left(2 \omega_{c},-\omega_{c}\right)}{\operatorname{Re}\left(\Gamma_{1}\left(2 \omega_{c}\right)\right)}\right. \\
& \left.-3 \Gamma_{3}\left(\omega_{c}, \omega_{c},-\omega_{c}\right)\right] .
\end{aligned}
$$

The first three relations involve the linear terms of both models. They are a consequence of the fact that, on the one hand, the coefficients of the linear terms of the GL equation depend on the dispersion relation $\omega(k, \mathrm{Re})$ and, on the other hand, the linear kernel of the Volterra model is proportional to the inverse dispersion relation $k(\omega, \mathrm{Re})$. The fourth relation fixes the value of the cubic nonlinear coupling in the GL equation in function of the nonlinear couplings of the Volterra model, in such a way as to make the two approaches compatible.

A Volterra model of the type (2) and the GL equation (8) are not always equivalent in the way expressed by Eqs. (27) to (30). They are if three hypotheses are satisfied: (1) the system is close to the linear instability threshold, (2) the dynamics is dominated by the marginal wave $\left(k_{c}, \omega_{c}\right)$, whose amplitude is a slowly varying function of time and space and (3) the first harmonic of the fundamental mode follows it adiabatically. These hypotheses are in any case those under which this amplitude equation can be derived. ${ }^{38}$

There is one more point to be underlined: from the beginning, we have neglected kernels of order higher than three in the Volterra series. While this is often imposed as a hypothesis that cannot be verified, the experiment clearly confirms such a truncation. Had 4th or 5th order kernels been important, then the terms generated by them would have led to a quintic GL equation and not to a cubic one.

\section{GINZBURG-LANDAU COEFFICIENTS FOR THE ROTATING DISK FLOW}

Now that a link has been established between the Volterra kernels and the coefficients of the GL equation, we discuss the inference of the latter from the experimental data.

Note that, in a strict sense, the results derived from the Volterra equation [Eqs. (19) and (21)] can be inserted into the GL equation [Eq. (12)] only if the spatial derivatives are taken in the same direction. This direction is given here by the propagation of the vortices, i.e., the group velocity $\partial \omega /\left.\partial \mathbf{k}\right|_{\mathbf{k}=\mathbf{k}_{\mathbf{c}}}$. Experimentally, however, we only have access to the spatial derivative of the flow $\partial v / \partial \mathbf{x}$ along a direction which is oblique to the group velocity; see Fig. 2. It should thus be kept in mind that we are dealing here with a "projected" Ginzburg-Landau equation. As for the validity of the adiabatic hypothesis (18), it can be checked on the experimental data, and is indeed satisfied.

We recall that the Volterra kernels contain pertinent physical information regardless of the model one is actually trying to fit. The imaginary part of the linear kernel $\Gamma_{1}(\omega)$, 


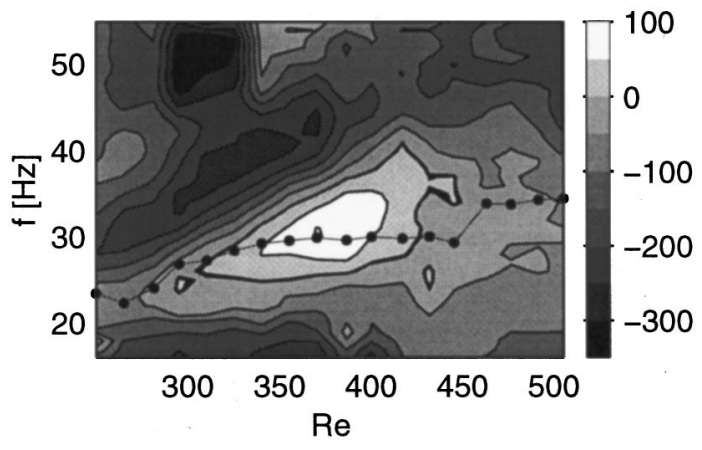

FIG. 5. The real part of the linear Volterra kernel $\Gamma_{1}$ for various frequencies and Reynolds numbers. Superimposed on it is the frequency $\omega_{c}$ of the fundamental.

for example, is directly related to the wavefield dispersion relation. We refer to Ref. 7 for a discussion on that. Likewise, the real part of $\Gamma_{1}(\omega)$ is related to the spatial linear growth rate. The real part $\operatorname{Re} \Gamma_{1}(\omega)$ is displayed in Fig. 5 together with the frequency $\omega_{c}$ of the fundamental mode.

Because of the lack of probe calibration, the value of the growth rate is known up to an additive constant. With the $a d$ hoc calibration we used, the uncertainty level is estimated to be about $50\left(\mathrm{~m}^{-1}\right)$ (i.e., one contour interval of Fig. 5). In spite of this, positive values of the growth rate confirm the onset of the instability around $\mathrm{Re}=300$, in good agreement with previous studies. ${ }^{7}$ As the Reynolds number increases, the growth rate reaches a maximum and then decays to become slightly negative. A plausible reason for this decay is the increasing difficulty in modeling the turbulent wavefield.

Using the values of Volterra kernels calculated from experimental data in Eqs. (27) to (30) we finally get, near the linear threshold $\left(\operatorname{Re}_{c} \approx 280\right)$,

$$
\begin{aligned}
& V_{g}=0.16 \pm 0.03 \mathrm{~m} \cdot \mathrm{s}^{-1}, \\
& \tau_{0}=15.1 \pm 3.2 \mathrm{~ms}, \\
& \xi_{0}=2.1 \pm 0.5 \mathrm{~mm}, \\
& c_{1}=-0.47 \pm 0.28 .
\end{aligned}
$$

These results agree with those obtained in Ref. 7. The relatively large uncertainty intervals reflect not only the fit of the Volterra kernels by the GL coefficients, but also the irrepro-

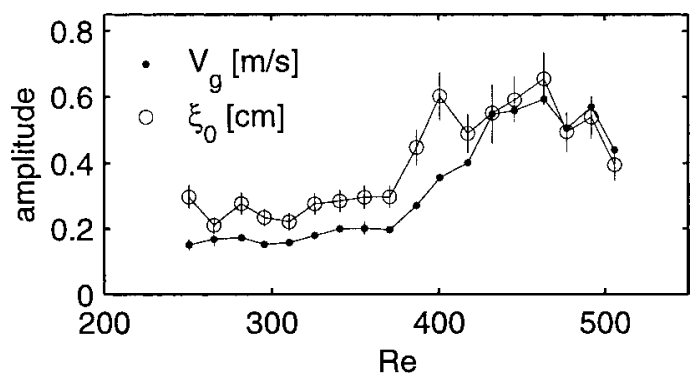

FIG. 6. Group velocity $V_{g}$ projected along the probe separation vector, and characteristic length $\xi$ as measured using the Volterra model, for different Reynolds numbers. Notice that they are expressed in different units.

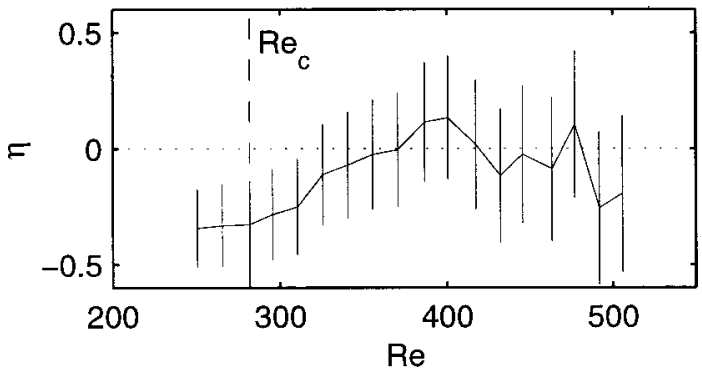

FIG. 7. Criterion for convective instability (31): the flow becomes absolutely unstable when $\eta$ is positive.

ducibility of the experiment, i.e., the difficulty in reproducing with a single model each pattern triggered by the roughness element. The error bars are obtained by fitting models with various numbers of kernels to different sequences of the time series. They are therefore conservative.

As far as the nonlinear part of the GL equation is concerned, namely the coefficients $c_{2}$ and $g$, our error bars are presently too large to meaningfully assess the value of these coefficients. There is strong evidence, however, for $g$ to be positive and $c_{2}$ negative. The absolute value of $g$ does not have any physical meaning in this particular application, since the probes measure a voltage and not a velocity. The positive sign of $g$, however, is important, as it confirms the supercritical nature of the bifurcation associated with the cross-flow instability.

The GL equation is in principle applicable only close to the linear instability threshold. However, its domain of validity is frequently extended to higher Reynolds numbers (up to secondary instabilities). In Fig. 6, we plot the values of the group velocity $V_{g}$ and diffusion length $\xi_{0}$ obtained in extrapolating formulas (27) and (29) for $\operatorname{Re}>\operatorname{Re}_{c}$. We cannot do this for $\tau_{0}$, because for $\operatorname{Re}>\operatorname{Re}_{c}$ the dependence of the growth rate of the fundamental mode from the Reynolds number is rapidly dominated by nonlinear effects: it is then meaningless to evaluate $\tau_{0}$ as the derivative of the linear growth rate with respect to Re.

In this figure, we see that the group velocity increases $V_{g}$ with Re, which is what one expects; ${ }^{7}$ the diffusion length $\xi_{0}$ does not vary much up in the range $280 \leqslant R e \leqslant 380$, and increases for $\operatorname{Re} \simeq 400$, as the wave packets triggered by the roughness element start to spread out and merge. The parameter $c_{1}$ does not vary significatively with the Reynolds numbers. Its value, which has no straightforward physical interpretation, is in agreement with that found in Ref. 7.

Let us finally consider the criterion of convective vs absolute instability of the flow. For $\operatorname{Re} \geqslant \operatorname{Re}_{c}$, let

$$
\eta=\frac{\operatorname{Re}-\operatorname{Re}_{c}}{\operatorname{Re}_{c}}-\frac{V_{g}^{2} \tau_{0}^{2}}{4 \xi_{0}^{2}\left(1+c_{1}^{2}\right)} .
$$

As it is discussed in Ref. 8, a flow is convectively unstable when $\eta$ is negative, and absolutely unstable when $\eta$ is positive. Figure 7 suggests that the transition to absolute instability takes place near $\operatorname{Re} \approx 400$, in agreement with the linear prediction given in Ref. 19 for a Rossby number estimated at $R_{0}=0.73$. This feature is also compatible with results ob- 
tained on the same data set, using demodulation techniques: in Ref. 20, it is shown that the spatial organization of the flow strongly increases at $\operatorname{Re} \simeq 380$, which might therefore correspond to the onset of absolute instability.

\section{CONCLUSIONS}

In this paper we present some properties of the flow in the boundary layer above a rotating disk. We describe the wavefield in terms of weakly interacting Fourier modes, using a Volterra series equation, whose kernels are estimated using two-point measurements of the wavefield.

One of the advantages of Volterra models is that they provide access to the nonlinear terms at various orders. Surprisingly, the dynamics of the flow is well described by a model containing a limited number (five to twelve) of linear and cubic kernels only. The prevalence of linear kernels is normal because we are in a weakly nonlinear regime. The quasi-absence of quadratic terms, whose manifestations are three-wave interactions, is more surprising. It is explained by the dispersion relation, which forbids three-wave resonances. Four-wave resonances, however, are not forbidden, and thus rule the nonlinear dynamics.

We have studied the relation of this model to the amplitude equation that is classically used to describe the instability of flows, the complex Ginzburg-Landau equation. We have shown that the two apparently different approaches are compatible. Analytical results for this are presented in detail: they are summarized by relations (27) to (30). These relations hold under the same hypotheses as those justifying the use of an amplitude equation, and can be applied to a large class of weakly nonlinear extended systems. Our results for the GL coefficients relative to the rotating disk flow are compatible with those obtained in Ref. 7.

Interestingly, the Volterra approach reveals that fourth and fifth order interactions are not significant, thereby jusfifying its truncation at the cubic term. This truncation is often imposed a priori as a hypothesis while here it is deduced from the experiment. Moreover, this confirms that our physical system can indeed be described by means of an amplitude equation with a cubic nonlinearity.

Thanks to their general structure and phenomenological character, Volterra equations can describe a wide variety of nonlinear behavior, while still offering a straightforward physical interpretation. That is why this analysis could be extended to different types of amplitude equations, for example to a quintic GL equation, ${ }^{40}$ including in this case higher order Volterra kernels in our model. This approach gives some insight in the structure and physical meaning of the different terms of amplitude equation considered, and can eventually be used to test if it really can apply to the system under study.

\section{A. Practical aspects of model identification}

In this section we briefly describe the numerical procedure for estimating the different kernels of the Volterra model, i.e., the coefficients of the nonlinear transfer function [Eq. (5)].

First, the transfer function is written in the matrix form

$$
y=P \Theta+e,
$$

with

$$
\mathrm{y}=\left[\begin{array}{c}
y_{1} \\
\vdots \\
y_{N}
\end{array}\right], \mathbf{P}=\left[\mathrm{p}_{1}, \ldots, \mathrm{p}_{M}\right], \Theta=\left[\begin{array}{c}
\theta_{1} \\
\vdots \\
\theta_{M}
\end{array}\right], \mathrm{e}=\left[\begin{array}{c}
\epsilon_{1} \\
\vdots \\
\epsilon_{N}
\end{array}\right] \text {. }
$$

The $M$ unknown parameters $\theta$ are the various kernels $\bar{g}$ of Eq. (5). The $N \times M$ matrix $\mathrm{P}$ (with $N>M$ to avoid underdetermination) contains the regressors $\mathrm{p}_{k}$, each of which is some combination of past and present inputs.

The minimization of the Euclidian norm $\|\mathrm{e}\|$ leads to a standard least squares problem that has a unique solution $\hat{\Theta}$. Various numerical methods are available for that purpose; see Ref. 36.

The ERR criterion proceeds as follows. Consider the Cholesky decomposition $\mathrm{P}$ into WA where $\mathrm{W}$ is an $N \times M$ orthogonal matrix and $\mathrm{A}$ is an $M \times M$ unit upper triangular matrix. The residuals can then be written as

$$
e=y-P \Theta=y-\left(P A^{-1}\right)(A \Theta)=y-W g .
$$

Equation (32) thus becomes

$$
\mathrm{y}=\mathrm{Wg}+\mathrm{e} .
$$

Computing the sum of squares of the output gives

$$
\mathrm{y}^{*} \mathrm{y}=\sum_{k=1}^{M} \mathrm{~g}_{k}^{2} \mathrm{w}_{k}^{*} \mathrm{w}_{k}+\mathrm{e}^{*} \mathrm{e}
$$

where * denotes complex conjugate transposition. The ERR is defined as the fraction of the output variance that is explained by each column vector $\mathrm{w}_{k}$,

$$
\operatorname{ERR}_{k}=\frac{\mathrm{g}_{i}^{2} \mathrm{w}_{k}^{*} \mathrm{w}_{k}}{\mathrm{y}^{*} \mathrm{y}}
$$

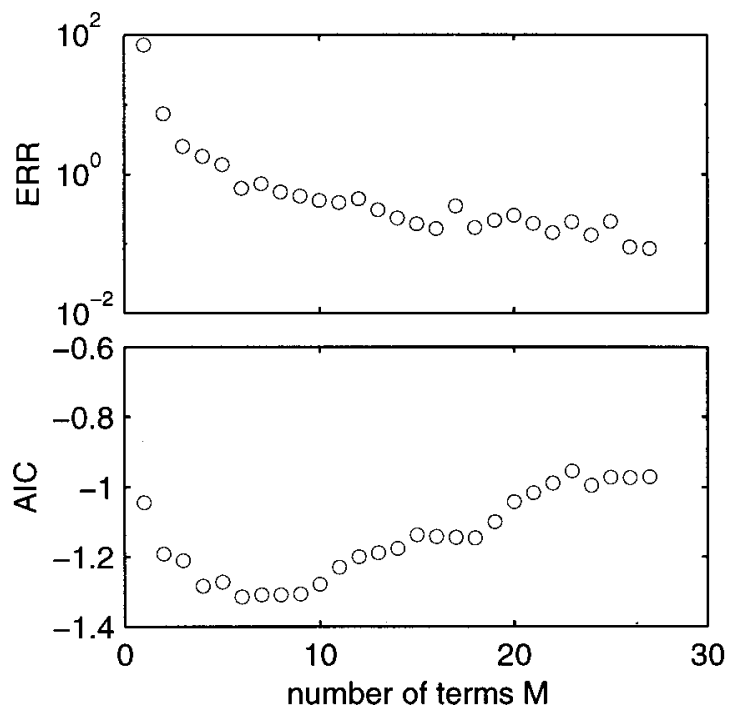

FIG. 8. Example of the evolution of the ERR criterion (top) and the AIC criterion (bottom) as the number of coefficients of the model increases. Notice that the ERR criterion is obtained using a single data set (the one used to estimate the kernels), whereas the AIC includes an additional stage of cross-validation, i.e., the model is first estimated and then tested using different data sets. 
with $0 \leqslant \mathrm{ERR} \leqslant 1$.

What we actually do is a Gram-Schmidt orthogonalization and the procedure goes as follows. First select the regressor $p_{k}$ whose correlation coefficient with the output $y$ is the largest. The corresponding unknown $\theta_{k}$ designates the first significant kernel. Then orthogonalize all regressors with respect to $\mathrm{p}_{k}$,

$$
\mathrm{p}_{\mathrm{i}}^{\prime}=\mathrm{p}_{\mathrm{i}}-\lambda \mathrm{p}_{k}, \forall i \neq k
$$

in order to have $\mathrm{p}_{i}^{*} \mathrm{p}_{k}=0$ if $i \neq k$. Find among the new regressors the one $\mathrm{p}_{\mathrm{m}}$ that has the strongest correlation with the output, and select the corresponding kernel. Then orthogonalize all regressors with respect to $\mathrm{p}_{\mathrm{m}}$ and repeat the procedure.

This algorithm is computationally efficient and requires little storage. Gram-Schmidt orthogonalization, however, is sensitive to round-off errors, but this is not a matter of concern since the number of needed kernels is reached long before round-off becomes a problem. Indeed, the ERR usually decreases fast enough to stop the procedure after less than a dozen steps. Depending on the noise level and the desired degree of accuracy, one typically wants the ERR to be about $10^{-2}$ to $10^{-5}$. Information theoretic criteria such as Akaike's Information Criterion (AIC) are further used to select the right number of coefficients. ${ }^{37}$

Figure 8 illustrates the gradual decrease of the ERR criterion for the rotating disk data. Round-off becomes significant here after the model has more than 20 coefficients. The figure also shows the AIC, which expresses the tradeoff between model complexity and ability to fit results. A global minimum is reached around 7 , which means that models containing more than 7 kernels do not contain significant new information. This is a safe indication for keeping 7 kernels only in the Volterra model.

Once the significant kernels have been identified, one solves the linear system

$$
\mathrm{y}=\mathrm{P}_{s} \Theta_{s}+\mathrm{e}
$$

in which the subscript ${ }_{s}$ means that we consider a subset only of the original system: the $N \times M_{s}$ matrix $\mathrm{P}_{s}$ contains only those regressors which correspond to significant kernels.

${ }^{1}$ N. Wiener, Nonlinear Problems in Random Theory (MIT Press, Cambridge, MA, 1958)

${ }^{2}$ M. Schetzen, The Volterra and Wiener Theories of Nonlinear Systems (Wiley, Chichester, NY, 1980).

${ }^{3}$ V. E. Zakharov, S. L. Musher, and A. M. Rubenchik, "Hamiltonian approach to the description of non-linear plasma phenomena," Phys. Rep. 129, 285 (1985).

${ }^{4}$ C. P. Ritz, E. J. Powers, and R. D. Bengston, "Experimental measurement of three-wave coupling and energy cascading," Phys. Fluids B 1, 153 (1989).

${ }^{5}$ T. Dudok de Wit, V. V. Krasnosel'skikh, M. Dunlop, and H. Lühr, “Identifying nonlinear wave interactions in space plasmas using two-point measurements: A case study of Short Large Amplitude Magnetic Structures (SLAMS),', J. Geophys. Res. 104, 17079 (1999).

${ }^{6}$ S. Jarre, P. Le Gal, and M. P. Chauve, "Experimental study of rotating disk flow instability. I. Natural flow," Phys. Fluids 8, 496 (1996).

${ }^{7}$ S. Jarre, P. Le Gal, and M. P. Chauve, "Experimental study of rotating disk flow instability. II. Forced flow," Phys. Fluids 8, 2985 (1996).

${ }^{8}$ R. J. Deissler, "Spatially growing waves, intermittency, and convective chaos in an open-flow system," Physica D 25, 233 (1987).
${ }^{9}$ H. T. Moon, P. Huerre, and L. G. Redekopp, "Transitions to chaos in the Ginzburg-Landau equation," Physica D 7, 135 (1983).

${ }^{10} \mathrm{~V}$. W. Ekman, "On the influence of the Earth's rotation on ocean currents," Ark. Mat., Astron. Fys. 2, 1 (1905).

${ }^{11}$ T. Von Karman, "Uber laminare und turbulente Reibung," Z. Angew. Math. Mech. 1, 233 (1921).

${ }^{12}$ A. J. Faller, "Instability and transition of disturbed flow over a rotating disk," J. Fluid Mech. 230, 245 (1991).

${ }^{13}$ A. J. Faller and R. E. Kaylor, "A numerical study of the instability of the laminar Ekman boundary layer," J. Atmos. Sci. 23, 466 (1966).

${ }^{14}$ N. Gregory, J. T. Stuart, and W. S. Walker, "On the stability of threedimensional boundary layers with application to the flow due to a rotating disk,' Philos. Trans. R. Soc. London 248, 155 (1955).

${ }^{15}$ R. Kobayashi, Y. Kohama, and Ch. Takadamate, "Spiral vortices in boundary layer transition regime on a rotating disk," Acta Mech. 35, 71 (1980).

${ }^{16}$ M. R. Malik, "The neutral curve for stationary disturbances in rotatingdisk flow," J. Fluid Mech. 164, 275 (1986).

${ }^{17}$ N. Aubry, R. Guyonnet, and R. Lima, "Spatio-temporal analysis of complex signals: Theory and applications," J. Stat. Phys. 64, 683 (1991).

${ }^{18}$ N. Aubry, R. Guyonnet, and M. P. Chauve, "Transition to turbulence on a rotating flat disk," Phys. Fluids 6, 2800 (1994).

${ }^{19}$ R. J. Lingwood, "Absolute instability of the Ekman layer and related rotating flows," J. Fluid Mech. 314, 373 (1995).

${ }^{20} \mathrm{P}$. Le Gal, "Complex demodulation applied to the transition to turbulence of the flow over a rotating disk," Phys. Fluids A 4, 2523 (1992).

${ }^{21}$ P. Le Gal, M. P. Chauve, L. Schouveiler, and S. Jarre, "Instabilités entre disque fixe et disque tournant," in Des Phénomènes Critiques au Chaos, colloque à la mémoire de P. Berge (CEA, Saclay, 1998).

${ }^{22} \mathrm{~V}$. Croquette and H. Williams, "Non-linear waves of the oscillatory instability of finite convective rolls," Physica D 37, 300 (1989).

${ }^{23}$ T. Leweke and M. Provansal, "Determination of the parameters of the Ginzburg-Landau wake model from experiments on a bluff ring," Europhys. Lett. 27, 655 (1994).

${ }^{24}$ J. M. Fullana, P. Le Gal, M. Rossi, and S. Zaleski, "Identification of parameters in an amplitude equation describing coupled wakes," Physica D 102, 35 (1997).

${ }^{25}$ D. P. Vallette, G. Jacobs, and J. P. Gollub, "Oscillations and spatiotemporal chaos of one-dimensional fluid fronts," Phys. Rev. E 55, 4274 (1997).

${ }^{26}$ J. F. Ravoux and P. Le Gal, "Bi-orthogonal decomposition analysis and reconstruction of spatio-temporal chaos generated by coupled wakes," Phys. Rev. E 58, R5233 (1998).

${ }^{27}$ M. B. Priestley, Non-Linear and Non-Stationary Time Series Analysis (Academic, London, 1988).

${ }^{28}$ A. S. Monin and A. M. Yaglom, Statistical Fluid Mechanics (MIT Press, Cambridge, MA, 1975), Vol. 2.

${ }^{29}$ D. R. Brillinger, "The identification of polynomial systems by means of higher order spectra,' J. Sound Vib. 12, 301 (1970).

${ }^{30}$ S. A. Billings, "Identification of nonlinear systems-A survey," Proc. IEE, Part D 127, 272 (1980).

${ }^{31}$ K. S. Lii, K. N. Helland, and M. Rosenblatt, "Estimating threedimensional energy transfer in isotropic turbulence," J. Time Ser. Anal. 3, 1 (1982).

${ }^{32}$ S. W. Nam and E. J. Powers, "Application of higher order spectral analysis to cubically nonlinear system identification," IEEE Trans. Acoust., Speech, Signal Process. ASSP-42, 1746 (1994).

${ }^{33} \mathrm{R}$. Haber and H. Unbehauen, "Structure identification of nonlinear dynamic systems-A survey on input/output approaches," Automatica 26, 651 (1990).

${ }^{34}$ J. Sjöberg, Q. Zhang, L. Ljung et al., "Nonlinear black-box modelling in system identification: A unified overview," Automatica 31, 1691 (1995).

${ }^{35}$ M. J. Korenberg, "Identification nonlinear difference equation and functional expansion representations: The fast orthogonal algorithm," Ann. Biomed. Eng. 16, 123 (1988).

${ }^{36}$ S. Chen, S. A. Billings, and W. Luo, "Orthogonal least squares methods and their application to non-linear system identification,' Int. J. Control 50, 1873 (1989).

${ }^{37}$ L. Ljung, System Identification, 2nd ed. (Prentice-Hall, Englewood Cliffs, NJ, 1999). 
${ }^{38} \mathrm{P}$. Manneville, Dissipative Structures and Weak Turbulence (Academic, New York, 1990).

${ }^{39} \mathrm{M}$. Gaster, "A note on the relation between temporally increasing and spatially increasing disturbances in hydrodynamic stability," J. Fluid
Mech. 14, 222 (1962).

${ }^{40}$ P. Jonas, B. Bruhn, B. P. Koch, and A. Dinklage, "Numerical investigations on strong pattern selecting Eckhaus instabilities in neon glow discharges,' Phys. Plasmas 7, 729 (2000). 